\title{
Impaired osteogenesis in Menkes disease- derived induced pluripotent stem cells
}

\author{
Dongkyu Kim', Jieun Choi ${ }^{1}$, Kyu-Min Han ${ }^{1}$, Beom Hee Lee ${ }^{2}$, Jin-Ho Choi ${ }^{2}$ Han-Wook Yoo ${ }^{2}$ and Yong-Mahn Han ${ }^{*}$
}

\begin{abstract}
Introduction: Bone abnormalities, one of the primary manifestations of Menkes disease (MD), include a weakened bone matrix and low mineral density. However, the molecular and cellular mechanisms underlying these bone defects are poorly understood.

Methods: We present in vitro modeling for impaired osteogenesis in MD using human induced pluripotent stem cells (iPSCs) with a mutated ATP7A gene. MD-iPSC lines were generated from two patients harboring different mutations.

Results: The MD-iPSCs showed a remarkable retardation in CD105 expression with morphological anomalies during development to mesenchymal stem cells (MSCs) compared with wild-type (WT)-iPSCs. Interestingly, although prolonged culture enhanced CD105 expression, mature MD-MSCs presented with low alkaline phosphatase activity, reduced calcium deposition in the extracellular matrix, and downregulated osteoblast-specific genes during osteoblast differentiation in vitro. Knockdown of ATP7A also impaired osteogenesis in WT-MSCs. Lysyl oxidase activity was also decreased in MD-MSCs during osteoblast differentiation.
\end{abstract}

Conclusions: Our findings indicate that ATP7A dysfunction contributes to retardation in MSC development and impairs osteogenesis in MD.

\section{Introduction}

Menkes disease (MD) is a copper metabolism disorder that is caused by a loss-of-function of a major copper transporter, ATP7A $[1,2]$. The ATP7A gene is located on the long arm of $\mathrm{X}$ chromosome and encodes a P-type ATPase, which plays crucial roles in cellular copper metabolism by controlling copper export and intracellular copper trafficking [3, 4]. Although its primary physiological function is copper absorption in the small intestine, ATP7A is also implicated in intracellular copper delivery to copper-dependent enzymes [5,6]. A variety of copper-dependent enzymes become nonfunctional due to a lack of ATP7A activity in MD patients, which can lead to multisystemic clinical symptoms $[6,7]$.

Clinical manifestations of MD patients include progressive neurodegeneration, connective tissue defects, sparse and kinky hairs, vascular defects, and many others. Connective tissue defects comprise tortuous

\footnotetext{
* Correspondence: ymhan57@kaist.ac.kr

${ }^{1}$ Department of Biological Science, Korea Advanced Institute of Science

Technology (KAIST), Daejeon 305-701, Republic of Korea

Full list of author information is available at the end of the article
}

vessels, skeletal change, loose skin, laxity of joints, and so forth [7-9]. Similarly, MD mouse models present with fragmentation of the internal elastic lamina, defective synthesis of bone collagen, reduced skin tensile strength, and weak blood vessels [10-12]. Among the various symptoms, bone abnormalities are a typical phenotype in MD patients [13-15]. Bone abnormalities in Menkes patients include osteoporosis, metaphyseal spurs, diaphyseal fractures, and wormian occipital bones [15-19]. Bone defects have also been shown in occipital horn syndrome, a mild phenotype of ATP7A-deficient disease [20]. Defective phenotypes in bone formation are frequently used as a diagnostic test in the early stage along with a blood test to measure low serum copper levels [16]. However, in vitro model systems that investigate how ATP7A mutations result in abnormal bone formation in MD have not been reported. Here, we attempt to model MD pathogenesis at the cellular level using induced pluripotent stem cells (iPSCs) in vitro. Human iPSCs, which have the capability to differentiate into various cell types and to proliferate indefinitely, are 
useful cell sources for studying the pathogenesis of human diseases [21, 22].

In this study, MD-iPSCs were differentiated into osteoblasts (OBs) to investigate the effect of ATP7A dysfunction on bone formation. Intriguingly, MD-iPSCs showed delayed mesenchymal stem cell (MSC) maturation compared with wild-type (WT)-iPSCs. Subsequently, MD-MSCs showed impaired osteogenesis in terms of alkaline phosphatase (ALP) activity, calcium mineralization, and transcription of osteogenic genes. Copper chelation in WT-MSCs resembled defective phenotypes shown in MD-MSCs. Our results demonstrate that dysfunction of copper utilization in MD gives rise to delayed MSC development and impaired OB differentiation.

\section{Materials and methods}

\section{Retrovirus production}

For retrovirus packaging, retroviral vectors encoding OCT4, SOX2, KLF4, cMYC (Addgene, Cambridge, MA, USA) were co-transfected with VSV-G vector (Takara Bio, Mountain View, CA, USA) in GP2 293 cells. Transfectants were further incubated in Dulbecco's modified Eagle's medium (DMEM; Welgene, Seoul, Korea) supplemented with $10 \%$ fetal bovine serum (FBS; Invitrogen, Carlsbad, CA, USA) and $1 \%$ penicillin-streptomycin (Invitrogen) at $37{ }^{\circ} \mathrm{C}, 5 \% \mathrm{CO}_{2}$ in air. The medium was changed $8 \mathrm{~h}$ after transfection. Then, supernatants were harvested 48 and $72 \mathrm{~h}$ after incubation. Supernatants harvested from four dishes $(10 \mathrm{~cm}$ in diameter) per factor were ultracentrifuged at $90,000 \times g$ for $90 \mathrm{~min}$ at $4{ }^{\circ} \mathrm{C}$. The viral pellet was dissolved in $2 \mathrm{ml}$ of the medium and kept at $-70{ }^{\circ} \mathrm{C}$ before use.

\section{Generation and maintenance of MD-iPSCs}

To generate MD-iPSCs, patient fibroblasts were infected with four retroviruses and then plated onto mitomycin C-treated (MMC; A.G. Scientific, San Diego, CA, USA) MEF feeder layers at a density of $10^{3}$ cells $/ \mathrm{cm}^{2}$. This study using patient fibroblasts was approved by the Institutional Review Board of Asan Medical Center, and written informed consent was obtained from their parents. Infected cells were cultured in human embryonic stem cell (ESC) medium at $37{ }^{\circ} \mathrm{C}, 5 \% \mathrm{CO}_{2}$ in air for 2 to 3 weeks. The human ESC medium consists of DMEM/ F12 (Invitrogen) supplemented with $20 \%$ Knockout SR (Invitrogen), $1 \%$ nonessential amino acids (Invitrogen), $1 \%$ penicillin-streptomycin, $0.1 \mathrm{mM} \beta$-mercaptoethanol (Sigma, St. Louis, MO, USA), and $10 \mathrm{ng} / \mathrm{ml}$ fibroblast growth factor (FGF)2 (R\&D systems, Minneapolis, MN, USA). Respective human ESC-like colonies were mechanically transferred onto new MMC-treated MEF feeders and subcultured for stabilization for 10 to 20 passages. iPSC characteristics were analyzed by the expression of human ESC markers, karyotypes, methylation states on promoters of human ESC marker genes, and teratoma formation. WT-iPSCs derived from foreskin fibroblasts [23] were used as a control group (Additional file 1: Figure S1).

\section{Real-time quantitative PCR}

Total mRNA was extracted from iPSCs and differentiated cells using easy-Blue ${ }^{\mathrm{mat}}$ (Intron Biotechnology, Seongnam, Korea). Briefly, approximately $1 \times 10^{5}$ cells were washed in phosphate-buffered saline (PBS) and treated with $1 \mathrm{ml}$ easy-Blue $^{\mathrm{Tu}}$ solution. After mixing with $200 \mu \mathrm{l}$ chloroform, cell lysates were centrifuged and the upper layer of the supernatants was harvested to isolate RNA. Then, RNA was precipitated and rehydrated for cDNA synthesis. A total of $1 \mu \mathrm{g}$ RNA was annealed with oligo(dT), and cDNA was synthesized using M-MLV Reverse Transcriptase (Enzynomics, Daejeon, Korea). The real-time polymerase chain reaction (RT-PCR) was performed using the following cycle conditions: $95^{\circ} \mathrm{C}$ denaturation, $60{ }^{\circ} \mathrm{C}$ annealing, and $72{ }^{\circ} \mathrm{C}$ elongation. The cycle numbers for each reaction varied between 30 and 40. Red safe (Intron Biotechnology) was used for visualization of PCR products in gel electrophoresis. For quantitative comparison, the relative expression level was measured by CFX-Connect real-time system (Bio-Rad, Hercules, CA, USA). The relative expression level of each gene was analyzed using a comparative threshold cycle method, and the transcription level of GAPDH was used for normalization. The primers used in this study are listed in Additional file 2 (Tables S1 and S2).

\section{Bisulfite sequencing}

Genomic DNA was isolated from cell samples using a G-DEX Genomic DNA Extraction Kit (Intron Biotechnology). Briefly, $2 \times 10^{6}$ cells were lysed in cell lysis buffer $(300 \mu \mathrm{l})$ at room temperature for $5 \mathrm{~min}$ and then incubated at $37^{\circ} \mathrm{C}$ for $30 \mathrm{~min}$ in the presence of RNase A. After the addition of PPT Buffer $(100 \mu \mathrm{l})$, cell lysates were centrifuged at $16,000 \times g$ for $5 \mathrm{~min}$, and the supernatant was harvested. Isopropanol $(300 \mu \mathrm{l})$ was added to the supernatant. After centrifugation at $16,000 \times g$ for $1 \mathrm{~min}$, the DNA pellet was dissolved in distilled water $(D W)$. Bisulfite treatment was performed using a Zymo EZ DNA methylation kit (Zymo Research, Irvine, CA, USA) according to manufacturer's instructions. Briefly, $1 \mu \mathrm{g}$ of genomic DNA was denatured at $95{ }^{\circ} \mathrm{C}$ for $10 \mathrm{~min}$, and CT-conversion was performed by addition of the CT Conversion Reagent. CT-converted DNA was desulfonated in M-Desulfonation Buffer, washed with M-Wash Buffer, and dissolved in $20 \mu \mathrm{l}$ of DW. Bisulfite-treated genomic DNA was amplified by $\mathrm{PCR}$, individually cloned into a $\mathrm{pGEM}^{\circ}-\mathrm{T}$ vector (Promega, Madison, WI, USA), and sequenced using an ABI 3730XL DNA Analyzer (Applied Biosystems, Foster City, 
CA, USA). Methylation quantification was performed using the QUMA program (Riken, Kobe, Japan). The primers used in bisulfate sequencing are listed in Additional file 2 (Table S3).

\section{Immunostaining}

The cells were fixed with $4 \%$ formaldehyde for $30 \mathrm{~min}$, washed twice in PBST (PBS containing $0.1 \%$ Tween 20), and permeabilized in PBS containing $0.1 \%$ Triton X-100 (Sigma) for $20 \mathrm{~min}$. After blocking with $2 \%$ bovine serum albumin (BSA; Sigma) for $1 \mathrm{~h}$, the cells were treated with each primary antibody and incubated at $4{ }^{\circ} \mathrm{C}$ overnight. The primary antibodies used in this study were as follows: OCT4 (Santa Cruz Biotechnology, Santa Cruz, CA, USA); SOX2 (Cell Signaling, Danvers, MA, USA); NANOG (R\&D Systems); SSEA4 (Abcam, Cambridge, England); TRA-1-60 (Millipore, Billerica, MA, USA); TRA-1-81 (Millipore); NESTIN (Millipore); $\alpha$-SMA (R\&D Systems); and GATA4 (Santa Cruz Biotechnology). The cells were washed several times in PBST and incubated with secondary antibodies (Alexa Fluor 488 or 594; Invitrogen) for $1 \mathrm{~h}$. Then, the cells were washed several times in PBST and counter-stained with 4'-6-diamidino-2-phenylindole (DAPI; Sigma) during the washing step. After washing with PBST, fluorescence images were observed on a Zeiss LSM 510 confocal microscope equipped with argon and helium-neon lasers (Carl Zeiss, Germany).

\section{Teratoma formation of MD-iPSCs}

Animal care and experimental procedures were performed under the approval of the Animal Care Committees of KAIST. MD-iPSCs $\left(1 \times 10^{7}\right.$ cells $)$ were collected by scraping, mixed with Matrigel (BD Biosciences, Franklin Lakes, NJ, USA), and subcutaneously injected into the dorso-lateral area of CAnN.Cg-Foxn1 nu/ CrljOri mice (Orient, Seongnam, Korea). Approximately 2 months after injection, the tumor tissues were dissected and embedded in paraffin wax. Tissue sections were placed on slide glasses. Hematoxylin and eosin (H\&E; Sigma) staining was performed to observe various cell types and tissues.

\section{Differentiation of human iPSCs into MSCs}

Differentiation of human iPSCs into MSCs was performed as previously described [24, 25]. Briefly, human iPSC colonies were mechanically dissected and transferred to low-adhesion petri dishes (SPL Lifesciences, Pocheon, Korea). Dissected human iPSCs spontaneously aggregated to form embryoid bodies (EBs) in EB medium at $37{ }^{\circ} \mathrm{C}$ with $5 \% \mathrm{CO}_{2}$ for 1 day. The EB medium consists of DMEM/F12 supplemented with $10 \%$ Knockout SR, 1 $\%$ nonessential amino acids, $1 \%$ penicillin-streptomycin, and $0.1 \mathrm{mM} \beta$-mercaptoethanol. EBs were further cultured in the EB medium containing $10 \mu \mathrm{M}$ SB431542
(Abcam) at $37{ }^{\circ} \mathrm{C}$ with $5 \% \mathrm{CO}_{2}$ for 10 days, and then attached to fibronectin-coated dishes (BD Biosciences). The attached cells were further cultured in DMEM/F12 supplemented with $1 \mu \mathrm{M}$ SB431542, 1 \% ITS Liquid media supplement (Sigma), 1 \% B27 supplement (Invitrogen), and $1 \% \mathrm{CD}$ lipid concentrate (Invitrogen) for 4 days. Then, the cells were cultured in $\alpha$-minimum essential medium ( $\alpha$-MEM; Invitrogen) containing $10 \%$ FBS for 20 days for MSC induction.

\section{FACS analysis}

Cells were dissociated by treatment with trypsin-EDTA ( $0.25 \%$; Invitrogen) for $5 \mathrm{~min}$ followed by the addition of fresh culture medium containing FBS for enzyme inactivation. After centrifugation at $300 \times g$ for $5 \mathrm{~min}$, the pellets were resuspended in FACS buffer (PBS containing $2 \%$ FBS) and filtered through a cell strainer with a $40-\mu \mathrm{m}$ pore size (SPL Lifesciences). Dissociated cells were incubated with specific FACS antibodies against CD44, CD73, CD90, CD105, and respective isotype controls (Biolegend, San Diego, CA, USA) at $4{ }^{\circ} \mathrm{C}$ for $30 \mathrm{~min}$. After washing with FACS buffer, samples were analyzed using a FACSCalibur flow cytometer (BD Biosciences). The positive population for each antibody was evaluated with Flowjo software (Tree Star, Ashland, OR, USA). Gating strategy for this analysis is summarized in Additional file 3 (Figure S2).

\section{Western blotting}

Cells were lysed with Pro-Prep protein extraction solution (Intron Biotechnology) on ice for $1 \mathrm{~h}$. After centrifugation at $16,000 \times g$ for $30 \mathrm{~min}$, the supernatant was harvested. The concentration of protein lysates was determined by Bradford protein assay (Bio-Rad). Proteins were loaded onto an SDS-PAGE gel (Elpis Biotech, Daejeon, Korea) and then transferred to a nitrocellulose membrane (Whatman, Maidstone, England). The membrane was blocked with TBST (0.1 \% Tween in TBS) containing 4 \% skim milk, washed in TBST, and treated with the appropriate primary antibodies. The primary antibodies used in this study were as follows: SMAD2 (Cell Signaling); p-SMAD2 (Cell Signaling); ACTIN (Santa Cruz Biotechnology); ATP7A (Hycult Biotech, Uden, Netherlands); SMAD1 (Cell Signaling); and p-SMAD1 (Cell Signaling). After washing in TBST, the membrane was incubated with an horseradish peroxidase (HRP)-conjugated secondary antibody (Thermo Fisher Scientific, Waltham, MA, USA) at room temperature for $1 \mathrm{~h}$. The membrane was developed using the ECL system (Thermo Fischer Scientific), and images were captured by LAS-3000 (Fuji Film, Tokyo, Japan).

\section{MSC proliferation and apoptosis}

Cells were seeded on a gelatin-coated dish at a density of $2 \times 10^{3}$ cells $/ \mathrm{cm}^{2}$ and cultured in $\alpha$-MEM containing $10 \%$ FBS for 9 days. The number of cells was 
calculated daily using a hemocytometer (Marienfeld, Lauda-Königshofen, Germany). To examine cell viability, cells were first plated on a gelatin-coated dish at a density of $2 \times 10^{4}$ cells $/ \mathrm{cm}^{2}$ and then cultured for 1 day. After the addition of the CCK- 8 reagents (Dojindo, Kumamoto, Japan), the cells were incubated at $37{ }^{\circ} \mathrm{C}$ for $30 \mathrm{~min}$, and absorbance was measured at $450 \mathrm{~nm}$. To detect apoptosis, cells were resuspended in $1 \mathrm{X}$ annexin $\mathrm{V}$ binding buffer (eBioscience, San Diego, CA, USA) and incubated with annexin V-FITC (eBioscience) and propidium iodide (PI; Sigma) for $15 \mathrm{~min}$ in the dark. After FACS analysis, annexin-positive cells were counted and graphed. For cell cycle analysis, cells were fixed in cold $70 \%$ ethanol for $1 \mathrm{~h}$ and then treated with RNase A (Sigma) for $30 \mathrm{~min}$. After treatment with PI for $15 \mathrm{~min}$, the distribution of cells in the cell cycle was analyzed on a FACSCalibur.

\section{MSC differentiation into OBs and chondrocytes}

For osteogenesis, MSCs were seeded on to gelatincoated dishes at a density of $2 \times 10^{4}$ cells $/ \mathrm{cm}^{2}$ and cultured in $\alpha$-MEM containing $10 \%$ FBS for 1 day. Then, MSCs were cultured in StemPro ${ }^{\circ}$ Osteogenesis Differentiation Medium (Invitrogen) at $37{ }^{\circ} \mathrm{C}$ with $5 \% \mathrm{CO}_{2}$ for 21 days. Medium was changed every 3-4 days. Differentiated cells were analyzed by ALP activity, alizarin red S staining, and Von Kossa staining. For chondrogenesis, MSCs were concentrated in $\alpha$-MEM containing $10 \%$ FBS at a density of $1 \times 10^{7}$ cells $/ \mathrm{ml}$. A $10 \mu \mathrm{l}$ droplet was placed into noncoated wells of a 96-well plate (SPL Lifesciences) for $1 \mathrm{~h}$, and $100 \mu \mathrm{l}$ StemPro ${ }^{\circ}$ Chondrogenesis Differentiation Medium (Invitrogen) was added to each well. The next day, the MSC spheroids that formed were further cultured in the same medium for 2-3 weeks. The medium was changed every 3-4 days. The Alcian blue staining method was utilized to confirm chondrogenic differentiation.

\section{ALP assay}

Fixative solution and ALP staining solution are required for the ALP assay. Fixative solution is a mixture of $25 \mathrm{ml}$ citrate solution (Sigma), $65 \mathrm{ml}$ acetone (Junsei Chemical, Tokyo, Japan) and $8 \mathrm{ml} 37 \%$ formaldehyde. To create the ALP staining solution, $1 \mathrm{ml}$ sodium nitrite solution (Sigma) was mixed with $1 \mathrm{ml}$ FBV solution (Sigma). After incubation at room temperature for $2 \mathrm{~min}, 45 \mathrm{ml}$ DW and $1 \mathrm{ml}$ naphthol As-BI alkaline solution (Sigma) were added to the mixture. For the ALP assay, the cells were fixed in fixative for $30 \mathrm{~s}$ and then incubated in ALP staining solution for $20 \mathrm{~min}$ in the dark.

\section{Alizarin red S, Von Kossa, and Alcian blue staining}

For alizarin red S staining, the cells were fixed with $10 \%$ formalin for $20 \mathrm{~min}$ and then incubated with alizarin red $\mathrm{S}$ staining solution (Millipore) for $20 \mathrm{~min}$. For Von Kossa staining, cells were fixed with $10 \%$ formalin for $20 \mathrm{~min}$ and then exposed to ultraviolet light in $5 \%$ silver nitrate (American Master Tech, Lodi, CA, USA) for $1 \mathrm{~h}$. After washing with DW, the cells were incubated in $5 \%$ sodium thiosulfate (American Master Tech) at room temperature for $3 \mathrm{~min}$ and then observed under an inverted microscope (Olympus, Tokyo, Japan). For Alcian blue staining, chondrogenic spheroids were fixed with $10 \%$ formalin for $30 \mathrm{~min}$ and embedded in $2 \%$ agarose (LPS solution, Seoul, Korea) in PBS. Sections of chondrogenic spheroids were treated with $3 \%$ acetic acid (Millipore) for 3 min and then incubated in Alcian blue staining solution (American Master Tech) for $30 \mathrm{~min}$. The stains were detected using an inverted microscope.

\section{Transfection of ATP7A-siRNA into WT-MSCs}

Predesigned siRNAs (Bioneer, Daejeon, Korea) targeting ATP7A were transfected into WT- MSCs using Lipofectamine ${ }^{\circ}$ RNAiMAX (Invitrogen). Briefly, a total of 5 pmol siRNA was diluted in $50 \mu \mathrm{l}$ Opti-MEM ${ }^{\circ}$ medum (Invitrogen) and mixed with $3 \mu$ l Lipofectamine ${ }^{\circ}$ RNAi- $^{-}$

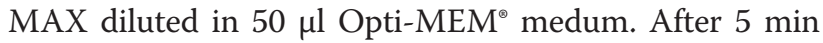
incubation at room temperature, siRNA-lipid complexes were added to the culture medium.

\section{Lysyl oxidase activity}

To measure lysyl oxidase (LOX) activity, supernatants were obtained 7 days after the induction of MSCs to OBs. The LOX assay reaction solution is a mixture of $20 \mu \mathrm{l} \mathrm{Amplite}{ }^{\mathrm{Tx}}$ HRP substrate stock solution (Abcam), $20 \mu \mathrm{l} \mathrm{HRP}(50 \mathrm{U} / \mathrm{ml}, \mathrm{Abcam})$, and $5 \mathrm{ml}$ assay buffer (Abcam). A total of $50 \mu \mathrm{l}$ supernatant and $50 \mu \mathrm{l} \mathrm{LOX}$ assay reaction solution were added to 96 wells. After incubation at $37^{\circ} \mathrm{C}$ for $20 \mathrm{~min}$ in the dark, fluorescence was measured at $\mathrm{Ex} / \mathrm{Em}=540 / 590$ using a microreader (Tecan, Maennedorf, Switzerland).

\section{Matrix collagen assay}

To measure collagen deposition in the extracellular matrix (ECM), human iPSC-derived OBs were fixed in $200 \mu \mathrm{l}$ Kahle fixative solution (Chondrex, Redmond, WA, USA) in four-well dishes for $10 \mathrm{~min}$. After washing with DW, the cells were incubated in $200 \mu \mathrm{l}$ dye solution (Chondrex) at room temperature for $30 \mathrm{~min}$. After removal of the dye solution, $500 \mu$ dye extraction buffer (Chondrex) was added to elute the bound dye solution. The OD values of the eluted dye solution were measured at $540 \mathrm{~nm}$ and $605 \mathrm{~nm}$ using a microreader.

\section{Measurement of intracellular copper concentration}

WT-MSCs and MD-MSCs were detached with trypsinEDTA and digested in a mixture of $2 \mathrm{ml} 65 \% \mathrm{HNO}_{3}$ (J.T. Baker ${ }^{\circ}, \mathrm{PA}, \mathrm{USA}$ ), and $7 \mathrm{ml}$ deionized water. Prepared samples were further digested in the Microwave 
Digestion System (Milestone Inc., CT, USA) for $4 \mathrm{~h}$ at $150{ }^{\circ} \mathrm{C}$. Then, copper concentration was analyzed by inductively coupled plasma (ICP) mass spectrometry (Agilent Technologies, CA, USA).

\section{Statistical analysis}

The statistical significance of the real-time RT-PCR data and other assays was evaluated by Student's $t$-test, and $p<0.05$ was considered significant.

\section{Results}

\section{Generation of iPSCs from MD patient fibroblasts}

Dermal fibroblasts were obtained from two different patients who each had a mutation in the ATP7A gene [26]. The 2-year-old Patient 1 (Menkes disease case 1, MD1) had an intronic mutation (c.4005 + 5G > A) that causes a splicing error on exon 20. The newborn Patient 2 (Menkes disease case 2, MD2) had a large genomic deletion (c.121-930_2626 + 488del) encompassing the exon 3-12 region (Table 1). The mutated region of patient MD1 is in the ATP-binding domain, which modulates the catalytic activity, and the deleted regions of MD2 include five copper-binding domains, four transmembrane regions, and fragments of a phosphatase domain that disturb a large portion of ATP7A (Additional file 4: Figure S3A). The two patients showed typical symptoms of MD, including severe neurodegeneration and intensive connective tissue abnormality (Table 1).

MD-iPSCs were generated from dermal fibroblasts of patients MD1 and MD2 by ectopic expression of OCT4, SOX2, cMYC, and KLF4. A MD1-iPSC clone, a MD2-iPSC clone, and WT-iPSCs were used in this study. MD1- and MD2-iPSCs had a typical morphology with tightly packed clusters and sharp boundaries and expressed pluripotency-associated marker genes (Fig. 1a and Additional file 4: Figure S3B). Exogenous genes were silenced after iPSC generation (Fig. 1b). MD1- and MD2-iPSCs differentiated into various cell types of the three germ layers in vitro (Additional file 4: Figure S3C) and formed teratomas after subcutaneous injection into nude mice (Fig. 1c). Furthermore, methylation of CpG dinucleotides in the promoter of OCT4, NANOG, REX1 genes were highly demethylated in MD1- and MD2iPSCs compared with each patient's fibroblasts (Fig. 1d), indicating successful epigenetic reprogramming. MD1iPSCs had a normal karyotype, and MD2-iPSCs showed a polymorphic variant (pericentric inversion of chromosome 9) that was the same as the karyotype of the MD2 patient (Additional file 4: Figure S3D). Mutations of the ATP7A gene were confirmed again at the genomic and transcriptional levels in MD1- and MD2-iPSCs (Additional file 4: Figure S3E and S3F).

\section{Differentiation of MD-iPSCs into MSCs}

MSCs were differentiated from MD-iPSCs using an EBbased method (Fig. 2a). In this method, EBs were treated with an inhibitor of transforming growth factor-beta signaling, SB431542 (SB), to enhance differentiation into cardiac mesoderm and neuro-ectoderm lineages. Treatment with SB efficiently blocked SMAD2 phosphorylation in all WT, MD1 and MD2 EBs (Additional file 5: Figure S4A). SB-treated EBs were morphologically normal in the three groups (Fig. 2b), and showed upregulated expression of a cardiac mesodermal gene, cTNT, and a neuroectodermal gene, NEUROD1, compared to undifferentiated cells (Additional file 5: Figure S4B). After attachment of SB-treated EBs to fibronectin-coated dishes, development of mesenchymal cells appeared to be retarded in MD-iPSCs (MD1- and MD2-iPSCs) compared with that of WT-iPSCs. Mesenchymal morphology could be

Table 1 Genetic information of Menkes patients

\begin{tabular}{|c|c|c|}
\hline Identification number & MD Case1 (MD1) & MD case2 (MD2) \\
\hline Gender & Male & Male \\
\hline Age at diagnosis & 4 months & 36 days \\
\hline Genotype & c.4005 + 5G > A of ATP7A (Splice site mutation) & c.121-930_2626 + 488del of ATP7A (Large deletion) \\
\hline Protein & Exon 20 deletion & Exon 3-12 deletion \\
\hline \multirow[t]{6}{*}{ Manifestations } & Lethargy & Diffuse cerebral dysfunction \\
\hline & Seizure & Developmental delay \\
\hline & Hypotonia & Hypotonia \\
\hline & Hypsarrhythmic pattern on EEG & Elongated tortuos intracranial vessels \\
\hline & Elongated tortuos intracranial vessels & Brittle hair \& loose skin \\
\hline & Brittle hair \& loose skin & \\
\hline Copper $(68-168 \mu \mathrm{g} / \mathrm{dl})$ & 17 & 8 \\
\hline Initial ceruloplasmin (13.1-42.8 mg/dl) & 4.6 & $<3$ \\
\hline Current outcomes & Death (4.7 years) & Bed ridden (2.6 years) \\
\hline
\end{tabular}


A

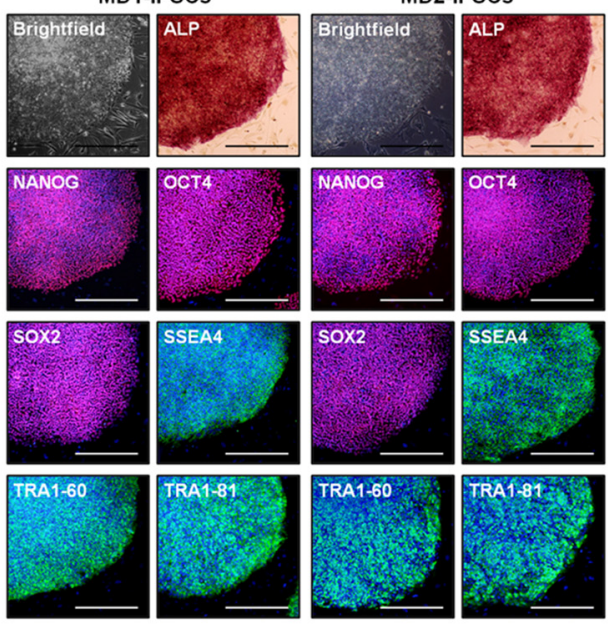

B

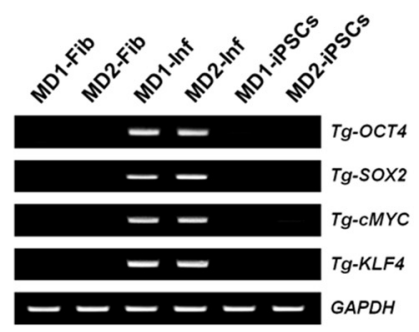

C

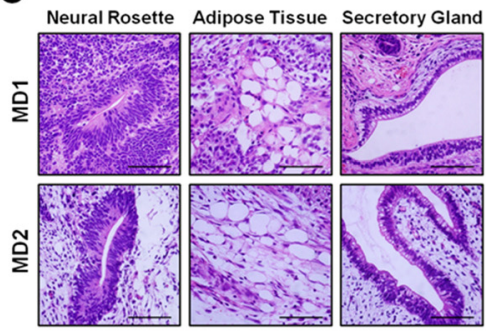

D

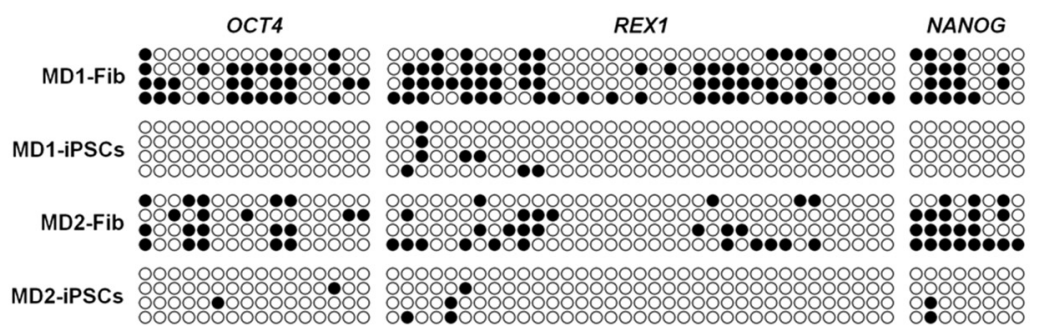

Fig. 1 Generation of MD-iPSCs. a Expression of pluripotent markers in MD-iPSCs. MD1- and MD2-iPSCs had normal morphologies and expressed pluripotent markers. Scale bars $=500 \mu \mathrm{m}$. b Transcriptional expression of transgenes such as OCT4, SOX2, CMYC, and KLF4 in MD-Fib, MD-inf, and MD-iPSCs. Transcription of the transgenes was detected only in infected MD1 and MD2 fibroblasts. c Teratoma formation of MD-iPSCs in immunodeficient mice. H\&E staining was performed to detect diverse cell types and tissues (neural rosette, ectoderm; adipose tissue, mesoderm; and secretory gland, endoderm). Scale bars $=100 \mu \mathrm{m}$. d Epigenetic reprogramming in MD-iPSCs. Promoters of pluripotent genes were highly demethylated in MD1- and MD2-iPSCs compared with fibroblasts. Each circle represents the methylation status of single CpG dinucleotides: empty circle, unmethylated; filled circle, methylated. ALP alkaline phosphatase, iPSC induced pluripotent stem cell, Inf infected fibroblasts, Fib normal fibroblasts, MD1/2 Menkes disease patient 1/2, Tg transgene

observed at 1 week after $\alpha$-MEM induction in the WTiPSC group, whereas mesenchymal morphology was observed at 2 weeks in the MD1- and MD2-iPSC groups (Fig. 2c). Differences in mesenchymal development between the WT- and MD-iPSC groups were also apparent after FACS analysis (Fig. 2d). CD105 expression in the MD1- and MD2-iPSC groups was relatively low by 3 weeks during mesenchymal development compared with the WT-iPSC group. The MD1- and MD2-iPSC groups also showed a slight reduction in CD90 expression after 1 week of $\alpha$-MEM induction, but no difference was detected in the expression of other MSC markers, such as CD44 and CD73, between the WT- and MD-iPSC groups. These results demonstrate that the induction of MD-EBs towards the mesenchyme may be delayed in the early stage. Intriguingly, however, MD1- and MD2MSCs achieved the normal MSC morphology and cell density of WT-MSCs after a long-term culture of 5 weeks (Fig. 3a). Furthermore, the expression level of
CD105 in MD-MSCs was similar to that of WT-MSCs (Fig. 3b), indicating the complete maturation of MSCs. In addition, mature MD-MSCs had normal cellular functions, including cell growth (Fig. 3c), viability (Fig. 3d), apoptosis (Fig. 3e) and cell cycle (Fig. 3f) compared with WT-MSCs. Thus, ATP7A mutations did not influence fundamental cellular functions in MSCs. Genetic mutations of the ATP7A gene were confirmed again in the MD1- and MD2-MSCs (Additional file 6: Figure S5A and S5B, respectively). The ATP7A protein was not detected in either the MD1- or MD2-MSCs (Additional file 6: Figure S5C), and MD-MSCs exhibited higher levels of intracellular copper than WTMSCs (Additional file 2: Table S4).

\section{Impaired osteogenesis of MD-MSCs}

To test the effect of ATP7A mutations on osteogenesis during bone formation, MD-MSCs were differentiated into OBs. To monitor OB differentiation, an ALP assay, 


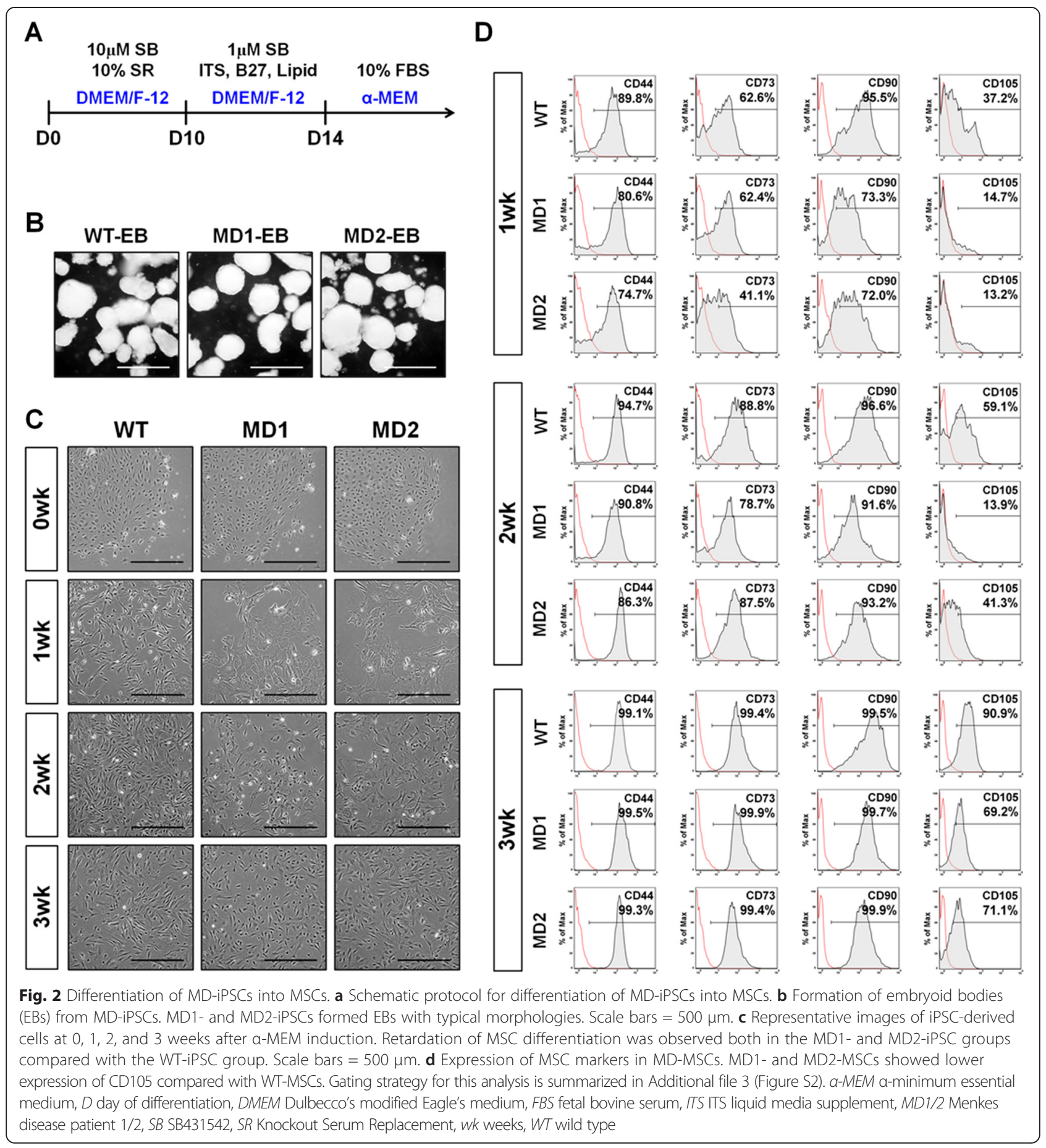

alizarin red S staining, and Von Kossa staining were performed. In WT-MSCs, ALP activity was clearly observed at 7 days and reached the highest level 14 days after $O B$ induction (Fig. 4a, upper panel). Intriguingly, ALP activity was barely detected at 7 days and was very weak even at 21 days in the MD1-MSCs during OB differentiation (Fig. 4a, middle panel). MD2-MSCs also showed low ALP activity in the process of OB differentiation (Fig. 4a, bottom panel). Thus, we found that MD-MSCs had aberrant ALP activity during OB differentiation. ALP activity is very important for calcium crystallization or mineralization during bone formation [27]. Therefore, we speculated that low ALP activity might lead to abnormal calcium deposition in MD-MSCs. As expected, MD1 - and MD2-MSCs showed lower levels of calcium deposition during $\mathrm{OB}$ differentiation compared with 


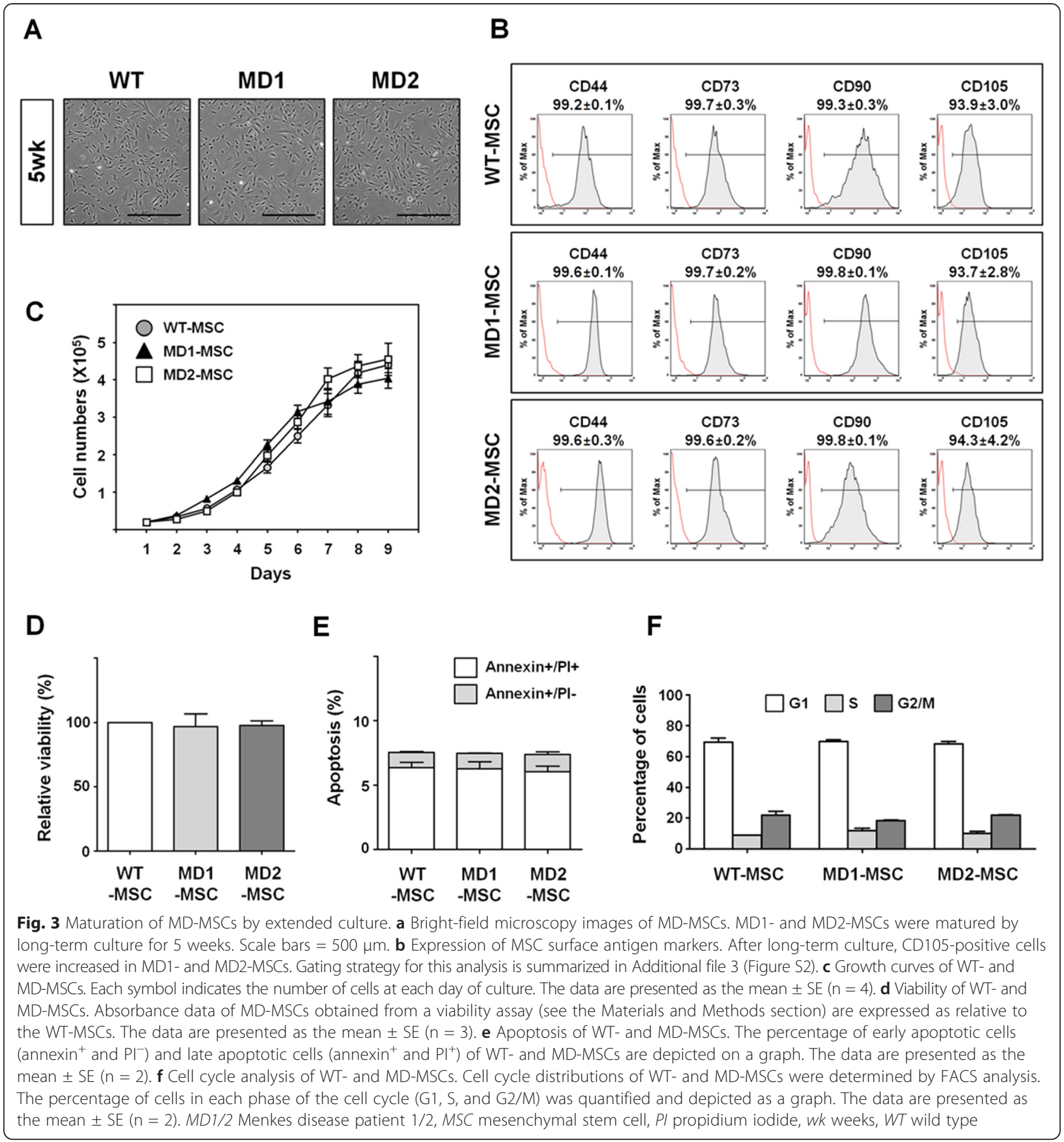

WT-MSCs (Fig. 4b and c). These results imply that reduced ALP activity accounts for insufficient calcium crystallization or mineralization. Relative expression of the matrix-related genes $O P N$ and $O C N$ was significantly downregulated, whereas the expression of the osteogenic transcription factor $R U N X 2$ was similar in MD-OBs compared with WT-OBs (Fig. 4d). To test whether ATP7A is associated with osteogenesis, a knockdown experiment using siRNA targeting ATP7A was carried out in WT-MSCs. In a preliminary experiment, siRNA use efficiently downregulated ATP7A transcript (Additional file 7: Figure S6). Knockdown of ATP7A in WT-MSCs also showed impaired osteogenesis with downregulation of osteogenic genes (Fig. 4e and f). Thus, knockdown of ATP7A in WT-MSCs recapitulated osteogenic impairments of MD-MSCs. Our results 
A
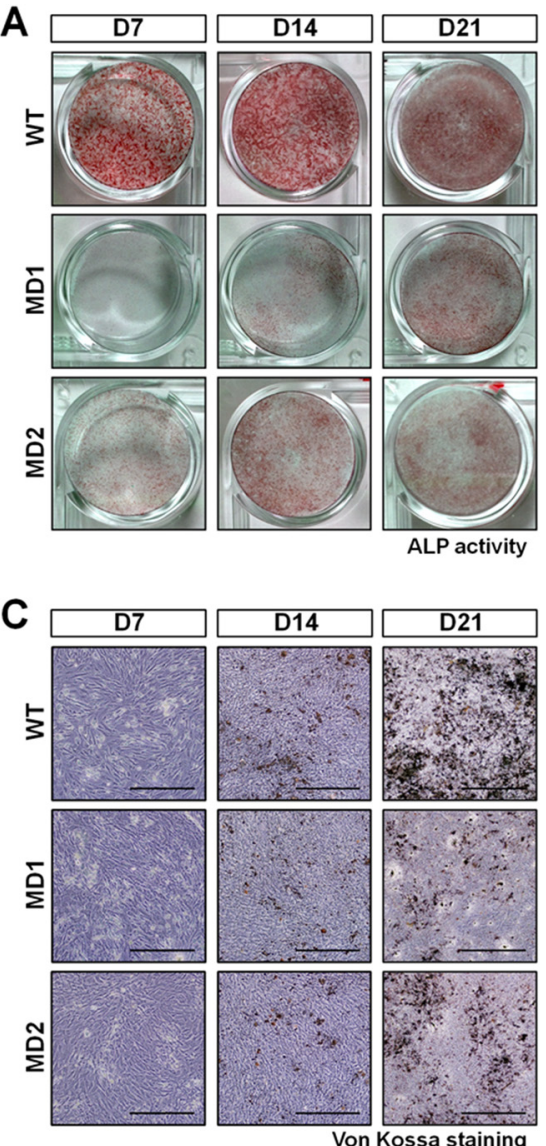

E

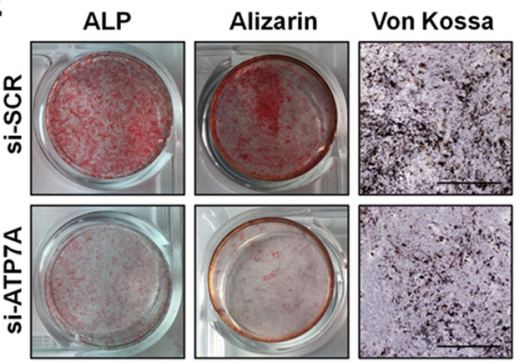

B

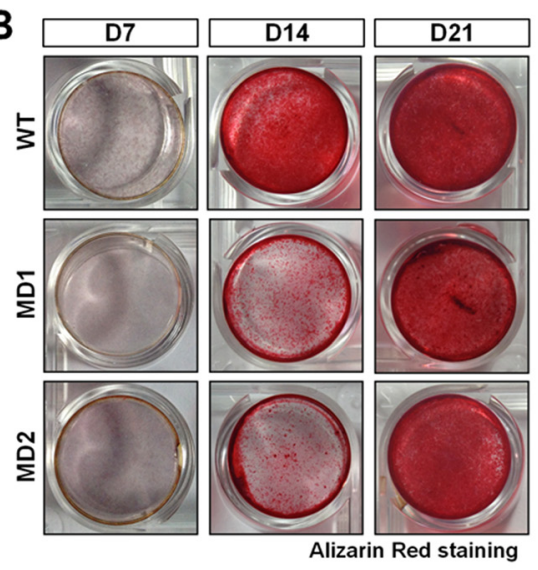

D

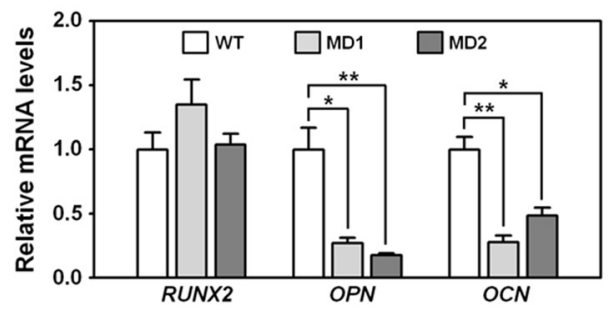

$\mathbf{F}$

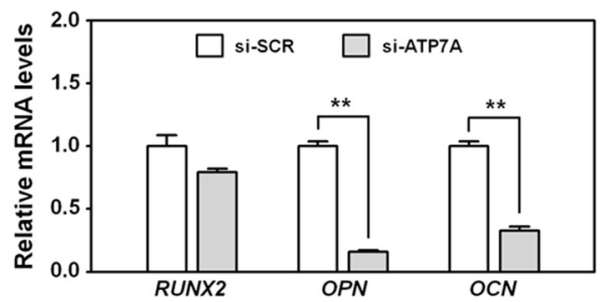

Fig. 4 Impaired osteogenesis in MD-MSCs. a Representative images of ALP activity in WT- and MD-MSCs during OB differentiation. ALP activity was observed as red granules. D, days after osteogenesis induction. $\mathbf{b}$ Representative images of alizarin red S staining in WT- and MD-MSCs during $\mathrm{OB}$ differentiation. Alizarin red S staining presented as red granules. c Representative images of Von Kossa staining in WT- and MD-MSCs during OB differentiation. Von Kossa staining was observed as black dots. Scale bars $=500 \mu \mathrm{m}$. d Relative expression of osteogenic genes RUNX2, OPN, and OCN in MD-MSCs during osteogenesis. The data are presented as the mean $\pm \mathrm{SE}(n=3)$. e, $\mathbf{f}$ Effects of ATP7A knock-down on osteogenesis in WT-MSCs. e Representative images of ALP activity, alizarin red S staining, and Von Kossa staining after transfection of siRNAs targeting ATP7A gene. Scramble siRNA (si-SCR) was also transfected in WT-MSCs as a control. f Relative expression of RUNX2, OPN, and OCN after ATP7A knockdown. The data are represented as the mean $\pm \mathrm{SE}(n=3) .{ }^{*} p<0.05,{ }^{* *} p<0.01$. ALP alkaline phosphatase, $D$ day of differentiation, MD1/2 Menkes disease patient 1/2, WT wild type

indicate that ATP7A plays an important role in bone formation.

Previous findings have shown that bone morphogenetic protein 2 (BMP2) induces OB differentiation of MSCs $[28,29]$. We therefore examined whether impaired osteogenesis in MD-MSCs is caused by insufficient activation of the BMP2 signaling pathway. No differences were detected in the activity of p-SMAD1 between the WT- and MD-MSCs (Additional file 8: Figure S7A). In addition, chondrogenesis appeared normal in MDMSCs (Additional file 8: Figure S7B). These results suggest that decrements of ALP activity and mineralization adversely affect osteogenesis in MD-MSCs. Next, activity of LOX, a copper-dependent enzyme, was measured 
to test whether it is associated with osteogenesis. The MD1- and MD2-OBs showed lower LOX activity than WT-OBs (Additional file 9: Figure S8A), but the amount of matrix collagen was not different between WT- and MD-OBs (Additional file 9: Figure S8B). Therefore, it is conceivable that impaired mineralization is not due to reduced deposition of collagen in MD-MSCs. Taken together, we suggest that the ATP7A mutation causes decreased ALP activity and mineralization, eventually resulting in impaired osteogenesis in MD.

\section{Discussion}

Here, we provide novel insight into the impaired osteogenesis in MD using iPSCs. ATP7A, which is a major copper transporter, plays important roles in copper absorption and delivery of copper to the human body $[6,8]$. Copper is one of the essential trace elements in normal development, and its homeostasis should be tightly regulated [30]. Disability of copper utilization in MD patients who have a defective ATP7A gene causes severe multisystemic phenotypes such as connective tissue abnormalities. In this study, MD-iPSCs with ATP7A mutations showed retardation of MSC development (Fig. 2d), although MD-MSCs matured after extended culturing in vitro (Fig. 3). Subsequently, several osteogenic defects, including decreased ALP activity and weak calcium mineralization, were observed in MD-MSCs and ATP7A-knockdown WT-MSCs during OB differentiation (Fig. 4).

ALP activity appears to be associated with calcium deposition during osteogenesis. During osteogenesis, ALP produces inorganic phosphate $(\mathrm{Pi})$ from pyrophosphate (PPi), and controls the balance between Pi and PPi levels in the ECM [31, 32]. Pi is further crystallized with calcium and accelerates calcium mineralization in the ECM. Dysfunction of the ALP gene causes a genetic disorder called hypophosphatasia, which is characterized by abnormal bone formation [27]. Here, low activity of ALP resulted in decreased calcium mineralization in MDMSCs during OB differentiation as shown by alizarin red $\mathrm{S}$ and Von Kossa staining (Fig. 4).

LOX mediates cross-linking of collagen and elastin in the ECM, which enhance tensile strength and structural integrity of connective tissues [3]. It has been postulated that decreased LOX activity accounts for the impaired connective tissue in MD patients [33, 34]. Reduced activity of LOX is also implicated in abnormal vasculopathy such as the vascular tortuosity and peripheral aneurysms in MD and its allelic variant, occipital horn syndrome (OHS) [35]. Aberrant internal elastic lamina structure is observed in the MD patient and the Menkes mouse model [36]. Cultured fibroblasts of MD and OHS show abnormalities in the expression of connective tissue genes [37]. Furthermore, bladder diverticula, inguinal hernia, skin laxity, hyperelasticity, and occipital exostosis are caused by reduced LOX activity [38].

In this study, LOX activity was decreased in MD-MSCs during $\mathrm{OB}$ differentiation. Nonetheless, there were no changes in collagen deposition in this study. These results raise the possibility that another role of LOX might be involvement in the aberrant $\mathrm{OB}$ differentiation in MDMSCs. In fact, it has been reported that LOX activity is involved in many biological functions other than collagen cross-linking, such as metastasis, tumor cell growth, cell migration and motility, angiogenesis, cell signaling, and transcription [39-42]. Thus, the identification of a new role for LOX in MD-MSCs during OB differentiation would be very interesting.

Taken together, these data show that utilization of intracellular copper is unavailable in MD cells due to dysfunctional ATP7A. This study provides additional insight into the pathophysiology of bone defects caused by the ATP7A mutation in MD.

\section{Conclusions}

Here we described the important role of ATP7A and copper during osteogenesis using MD-derived iPSCs. During OB differentiation, several osteogenic impairments such as low ALP activity, reduced calcium mineralization and decreased expression of osteogenic marker genes were observed. Knockdown of ATP7A in WT-MSCs recapitulated the impaired osteogenesis observed in MD-MSCs. Our results provide new insight into the important role of ATP7A in bone formation.

\section{Additional files}

Additional file 1: Figure S1. Characterization of WT-PSCS. (A) Expression of pluripotent markers in WT-iPSCs. (B) Teratoma formation of MD-iPSCs in immunodeficient mice. (C) Epigenetic reprogramming in WT-iPSCs. (TIFF $2486 \mathrm{~kb}$ )

Additional file 2: Table S1. Primers used in RT-PCR analysis. Table S2 Primers used in ATP7A genotyping of MD-derived cells. Table S3 Primers used in methylation analysis. Table S4 Copper concentration in WT- and MD-MSCs. (DOCX $19 \mathrm{~kb}$ )

Additional file 3: Figure S2. Detailed gating strategy for each MSC surface antigens. Gating strategy for CD44 surface antigen shown as a representative. Live cells (gate A) were gated based on forward scatter and side scatter. Staining with isotype control (PE-conjugated) was used to exclude CD44-negative events. This gate was then applied to samples stained with anti-CD44 antibody (PE-conjugated) to identify CD44 positive events. In merged image, red line indicates isotype control and black tinted area indicates CD44 positive events. (TIFF $1455 \mathrm{~kb}$ )

Additional file 4: Figure S3. Characterization of MD-iPSCs. (A) Schematic defective regions of ATP7A in MD1 and MD2 patients. The functional domains of ATP7A are depicted. ATP7A has six copper-binding domains, one phosphatase domain, one phosphorylation domain, one ATP-binding domain, and eight transmembrane domains. The defective regions of each patient are marked as boxes. (B) Transcriptional expression of pluripotent genes in MD-fibroblasts and MD-iPSCs. GAPDH was used as a control. (C) In vitro differentiation of MD-iPSCs. Immunostaining of NESTIN (ectoderm, red), a-SMA (mesoderm, green), and GATA4 (endoderm, green) was performed 7 days after spontaneous EB differentiation. DAPI showed nuclear counterstaining 
(blue). Scale bar $=500 \mu \mathrm{m}$. (D) Karyotypes of MD1- and MD2-iPSCs. (E) Genetic mutations in MD1-fibroblasts and MD1-iPSCs. A single-base substitution was confirmed in MD1-fibroblasts and MD1-iPSCs (Figure E-a). The resultant PCR products were different in MD1-fibroblasts and MD1-PSSCs (Figure E-b). The size difference (450 bp in WT and $246 \mathrm{bp}$ in MD1) generated by exon skipping was analyzed by gel electrophoresis. (F) Mutations in MD2-fibroblasts and MD2-iPSCs. Strategy for duplex PCR was explained as an illustration (Figure F-a). Size differences (532 bp in WT and 224 bp in MD2) generated by a large genomic deletion between WT- and MD2-fibroblasts were detected (Figure F-b). The primers used in this study are listed in Additional file 1 (Table S2). (TIFF $4321 \mathrm{~kb}$ )

Additional file 5: Figure S4. Characterization of EBs during MSC differentiation. (A) SMAD2 phosphorylation in WT- and MD-EBs during MSC differentiation. SB treatment suppressed p-SMAD2 in WT- and MD-EBs. (B) Relative expression of neuro-ectoderm and cardiac mesoderm marker genes (NEUROD1 and CTNT, respectively) in WT- and MD-EBs. The data are presented as the mean \pm SE $(n=3) ;{ }^{*} p<0.05,{ }^{* *} p<0.01$. (TIFF $2052 \mathrm{~kb}$ )

Additional file 6: Figure S5. Confirmation of MD-MSCs. (A) Genetic mutation of the ATP7A gene in MD1-MSCs. A single-base substitution was confirmed again in MD1-MSCs. (B) Genetic mutation of the ATP7A gene in MD2-MSCs. Size differences in the PCR product were observed in MD2-MSCs. (C) Expression of ATP7A in WT- and MD-MSCs. ATP7A protein was not detected in MD1- and MD2-MSCs. (TIFF $936 \mathrm{~kb}$ )

Additional file 7: Figure S6. Relative expression of ATP7A after siRNA transfection. The data are represented as the mean \pm SE $(n=3) ;{ }^{* *} p<0.01$. (TIFF $326 \mathrm{~kb}$ )

Additional file 8: Figure S7. Osteogenesis and chondrogenesis from MSCs. (A) SMAD1 phosphorylation in WT- and MD-MSCs during osteogenesis. (B) Differentiation of WT- and MD-MSCs into chondrocytes. WT- and MD-MSCs differentiated into chondrocytes following the procedures described in the Materials and Methods section. Morphologies of chondrocyte spheroids were similar between WT- and MD-MSCs (upper panel). Scale bar $=500 \mu \mathrm{m}$. Additionally, MD-MSCs stained normally with Alcian blue solution (lower panel). Scale bar = 50 m. (TIFF $1998 \mathrm{~kb}$ )

Additional file 9: Figure S8. Activity of LOX and matrix collagen in WT- and MD-OBs. (A) LOX activity in WT- and MD-OBs. Fluorescence data showing the LOX activity of MD-OBs (see the Materials and Methods section) are expressed as values relative to those of WT-OBs. The data are presented as the mean $\pm \mathrm{SE}(n=3) ;{ }^{*} p<0.05,{ }^{* *} p<0.01$ (B) Relative amount of matrix collagen in WT- and MD-OBs. The absorbance data obtained from a matrix collagen assay (see the Materials and Methods section) are expressed as values relative to that of WT-OBs. The data are presented as the mean \pm SE $(n=3)$. (TIFF $813 \mathrm{~kb})$

\section{Abbreviations}

ALP: Alkaline phosphatase; a-MEM: a-Minimum essential medium; BMP2: Bone morphogenetic protein 2; BSA: Bovine serum albumin; DAPI: 4'-6-diamidino-2-phenylindole; DMEM: Dulbecco's modified Eagle's medium; DW: Distilled water; EBs: Embryoid bodies; ECM: Extracellular matrix; ESC: Embryonic stem cell; FBS: Fetal bovine serum; FGF: Fibroblast growth factor; H\&E: Hematoxylin and eosin; HRP: Horseradish peroxidase; ICP: Inductively coupled plasma; iPSC: Induced pluripotent stem cell; LOX: Lysyl oxidase; MD: Menkes disease; MMC: Mitomycin C; MSC: Mesenchymal stem cell; OB: Osteoblast; OHS: Occipital horn syndrome; PBS: Phosphate-buffered saline; PBST: Phosphate-buffered saline + $0.1 \%$ Tween 20; Pi: Inorganic phosphate; PI: Propidium iodide; PPI: Pyrophosphate; RT-PCR: Real-time polymerase chain reaction; SB: SB431542; WT: Wild type.

\section{Competing interests}

The authors declare that they have no competing interests.

\section{Authors' contributions}

DK designed the study, carried out disease modeling, interpreted data and drafted the manuscript. JC carried out differentiation study, interpreted data and helped to revise the manuscript. KMH performed the characterization study, interpreted data, and drafted the manuscript. BHL studied patient samples, interpreted data, and helped to draft the manuscript. JHC participated in genetic analysis and helped to revise the manuscript. HWY helped to design the study and drafted the manuscript. YMH managed the project, designed the study, interpreted data, and drafted the manuscript. All authors read and approved the final manuscript.

\section{Acknowledgements}

We thank Ms. Sora Oh for the maintenance of human iPSCs. This work was supported by the NRF Stem Cell Program (2011-0019509) funded by MSIP and a grant (A120275) from Korea Healthcare technology R\&D project, MHW, Republic of Korea.

\section{Author details}

'Department of Biological Science, Korea Advanced Institute of Science Technology (KAIST), Daejeon 305-701, Republic of Korea. ${ }^{2}$ Department of Pediatrics, Asan Medical Center Children's Hospital, University of Ulsan College of Medicine, Seoul, South Korea.

Received: 22 January 2015 Revised: 30 April 2015

Accepted: 5 August 2015 Published online: 07 September 2015

\section{References}

1. Danks DM, Campbell PE, Stevens BJ, Mayne V, Cartwright E. Menkes's kinky hair syndrome. An inherited defect in copper absorption with widespread effects. Pediatrics. 1972;50:188-201.

2. Kaler SG. Diagnosis and therapy of Menkes syndrome, a genetic form of copper deficiency. Am J Clin Nutr. 1998;67:1029S-34.

3. La Fontaine S, Mercer JF. Trafficking of the copper-ATPases, ATP7A and ATP7B: role in copper homeostasis. Arch Biochem Biophys. 2007;463:149-67. doi:10.1016/j.abb.2007.04.021.

4. Barry AN, Shinde U, Lutsenko S. Structural organization of human Cu-transporting ATPases: learning from building blocks. J Biol Inorg Chem. 2010;15:47-59. doi:10.1007/s00775-009-0595-4.

5. Monty JF, Llanos RM, Mercer JF, Kramer DR. Copper exposure induces trafficking of the menkes protein in intestinal epithelium of ATP7A transgenic mice. J Nutr. 2005;135:2762-6.

6. Lutsenko S, Barnes NL, Bartee MY, Dmitriev OY. Function and regulation of human copper-transporting ATPases. Physiol Rev. 2007;87:1011-46. doi:10.1152/physrev.00004.2006.

7. Kaler SG. ATP7A-related copper transport diseases-emerging concepts and future trends. Nat Rev Neurol. 2011;7:15-29. doi:10.1038/nrneurol.2010.180.

8. de Bie P, Muller P, Wijmenga C, Klomp LW. Molecular pathogenesis of Wilson and Menkes disease: correlation of mutations with molecular defects and disease phenotypes. J Med Genet. 2007;44:673-88. doi:10.1136/jmg.2007.052746.

9. Tumer Z, Horn N. Menkes disease: recent advances and new aspects. J Med Genet. 1997;34:265-74.

10. Hunt DM. Primary defect in copper transport underlies mottled mutants in the mouse. Nature. 1974;249:852-4.

11. Mercer JF. Menkes syndrome and animal models. Am J Clin Nutr. 1998;67:1022S-8.

12. Rowe DW, McGoodwin EB, Martin GR, Grahn D. Decreased lysyl oxidase activity in the aneurysm-prone, mottled mouse. J Biol Chem. 1977;252:939-42.

13. Kreuder J, Otten A, Fuder H, Tumer Z, Tonnesen T, Horn N, et al. Clinical and biochemical consequences of copper-histidine therapy in Menkes disease. Eur J Pediatr. 1993;152:828-32.

14. Danks DM, Campbell PE, Walker-Smith J, Stevens BJ, Gillespie JM, Blomfield J, et al. Menkes' kinky-hair syndrome. Lancet. 1972;1:1100-2.

15. Nassogne MC, Sharrard M, Hertz-Pannier L, Armengaud D, Touati G, Delonlay-Debeney $\mathrm{P}$, et al. Massive subdural haematomas in Menkes disease mimicking shaken baby syndrome. Childs Nerv Syst. 2002;18:729-31. doi:10.1007/s00381-002-0630-z.

16. Amador E, Domene R, Fuentes C, Carreno JC, Enriquez G. Long-term skeletal findings in Menkes disease. Pediatr Radiol. 2010;40:1426-9. doi:10.1007/s00247-010-1551-8.

17. Kanumakala S, Boneh A, Zacharin M. Pamidronate treatment improves bone mineral density in children with Menkes disease. J Inherit Metab Dis. 2002:25:391-8. doi:10.1023/A:1020103901969.

18. Moller LB, Lenartowicz M, Zabot MT, Josiane A, Burglen L, Bennett C, et al. Clinical expression of Menkes disease in females with normal karyotype. Orphanet J Rare Dis. 2012;7:6. doi:10.1186/1750-1172-7-6. 
19. Baerlocher KE, Steinmann B, Rao VH, Gitzelmann R, Horn N. Menkes disease - clinical, therapeutic and biochemical studies. J Inherit Metab Dis. 1983;6:87-8. doi:10.1007/Bf01810339.

20. Kaler SG. Metabolic and molecular bases of Menkes disease and occipital horn syndrome. Pediatr Dev Pathol. 1998;1:85-98.

21. Grskovic M, Javaherian A, Strulovici B, Daley GQ. Induced pluripotent stem cells_opportunities for disease modelling and drug discovery. Nat Rev Drug Discov. 2011;10:915-29. doi:10.1038/nrd3577.

22. Maury Y, Gauthier M, Peschanski M, Martinat C. Human pluripotent stem cells for disease modelling and drug screening. Bioessays. 2012;34:61-71. doi:10.1002/bies.201100071.

23. Kim H, Kim D, Jang MJ, Han YM. Variations in the epigenetic regulation of lineage-specific genes among human pluripotent stem cell lines. Biochem Biophys Res Commun. 2012;424:331-7. doi:10.1016/j.bbrc.2012.06.122.

24. Mahmood A, Harkness L, Schroder HD, Abdallah BM, Kassem M. Enhanced differentiation of human embryonic stem cells to mesenchymal progenitors by inhibition of TGF-beta/activin/nodal signaling using SB-431542. J Bone Miner Res. 2010;25:1216-33. doi:10.1002/jbmr.34

25. Tran NT, Trinh QM, Lee GM, Han YM. Efficient differentiation of human pluripotent stem cells into mesenchymal stem cells by modulating intracellular signaling pathways in a feeder/serum-free system. Stem Cells Dev. 2012;21:1165-75. doi:10.1089/scd.2011.0346.

26. Kim JH, Lee BH, Kim YM, Choi JH, Kim GH, Cheon CK, et al. Novel mutations and clinical outcomes of copper-histidine therapy in Menkes disease patients. Metab Brain Dis. 2014;30:75-81. doi:10.1007/s11011-014-9569-5.

27. Whyte MP. Hypophosphatasia and the role of alkaline phosphatase in skeletal mineralization. Endocr Rev. 1994;15:439-61. doi:10.1210/edrv-15-4-439.

28. Gersbach CA, Guldberg RE, Garcia AJ. In vitro and in vivo osteoblastic differentiation of BMP-2- and Runx2-engineered skeletal myoblasts. J Cell Biochem. 2007;100:1324-36. doi:10.1002/jcb.21118.

29. Chen D, Harris MA, Rossini G, Dunstan CR, Dallas SL, Feng JQ, et al. Bone morphogenetic protein 2 (BMP-2) enhances BMP-3, BMP-4, and bone cell differentiation marker gene expression during the induction of mineralized bone matrix formation in cultures of fetal rat calvarial osteoblasts. Calcif Tissue Int. 1997:60:283-90.

30. Festa RA, Thiele DJ. Copper: an essential metal in biology. Curr Biol. 2011:21:R877-83. doi:10.1016/j.cub.2011.09.040

31. Murshed M, Harmey D, Millan JL, McKee MD, Karsenty G. Unique coexpression in osteoblasts of broadly expressed genes accounts for the spatial restriction of ECM mineralization to bone. Genes Dev. 2005;19:1093-104. doi:10.1101/gad.1276205.

32. Russell RG, Bisaz S, Donath A, Morgan DB, Fleisch H. Inorganic pyrophosphate in plasma in normal persons and in patients with hypophosphatasia osteogenesis imperfecta, and other disorders of bone. J Clin Invest. 1971;50:961-9. doi:10.1172/JCl106589.

33. Kagan HM, Li WD. Lysyl oxidase: Properties, specificity, and biological roles inside and outside of the cell. J Cell Biochem. 2003;88:660-72. doi:10.1002/Jcb.10413.

34. Lucero HA, Kagan HM. Lysyl oxidase: an oxidative enzyme and effector of cell function. Cell Mol Life Sci. 2006:63:2304-16. doi:10.1007/s00018-006-6149-9.

35. Godwin SC, Shawker T, Chang B, Kaler SG. Brachial artery aneurysms in Menkes disease. J Pediatr. 2006;149:412-5. doi:10.1016/j.jpeds.2006.05.041.

36. Murakami $\mathrm{H}$, Kodama $\mathrm{H}$, Nemoto $\mathrm{N}$. Abnormality of vascular elastic fibers in the macular mouse and a patient with Menkes' disease: ultrastructural and immunohistochemical study. Med Electron Microsc. 2002;35:24-30. doi:10.1007/s007950200003

37. Kemppainen R, Hamalainen ER, Kuivaniemi H, Tromp G, Pihlajaniemi T, Kivirikko KI. Expression of mRNAs for lysyl oxidase and type III procollagen in cultured fibroblasts from patients with the menkes and occipital horn syndromes as determined by quantitative polymerase chain reaction. Arch Biochem Biophys. 1996:328:101-6. doi:10.1006/abbi.1996.0148.

38. Kuivaniemi H, Peltonen L, Palotie A, Kaitila I, Kivirikko Kl. Abnormal copper-metabolism and deficient lysyl oxidase activity in a heritable connective-tissue disorder. J Clin Investig. 1982;69:730-3. doi:10.1172/Jci110503.

39. Rodriguez C, Martinez-Gonzalez J, Raposo B, Alcudia JF, Guadall A, Badimon L. Regulation of lysyl oxidase in vascular cells: lysyl oxidase as a new player in cardiovascular diseases. Cardiovasc Res. 2008;79:7-13. doi:10.1093/cvr/cvn102.
40. Baker AM, Cox TR, Bird D, Lang G, Murray Gl, Sun XF, et al. The role of lysyl oxidase in SRC-dependent proliferation and metastasis of colorectal cancer J Natl Cancer Inst. 2011;103:407-24. doi:10.1093/jnci/dja569.

41. Baker AM, Bird D, Welti JC, Gourlaouen M, Lang G, Murray Gl, et al. Lysyl oxidase plays a critical role in endothelial cell stimulation to drive tumor angiogenesis. Cancer Res. 2013;73:583-94. doi:10.1158/0008-5472.CAN-12-2447.

42. Erler JT, Giaccia AJ. Lysyl oxidase mediates hypoxic control of metastasis. Cancer Res. 2006;66:10238-41. doi:10.1158/0008-5472.CAN-06-3197.

\section{Submit your next manuscript to BioMed Central and take full advantage of:}

- Convenient online submission

- Thorough peer review

- No space constraints or color figure charges

- Immediate publication on acceptance

- Inclusion in PubMed, CAS, Scopus and Google Scholar

- Research which is freely available for redistribution 